The ground state of $C r$ II is $\mathrm{a}^{6} \mathrm{~S}$, the forbidden multiplet $\mathrm{a}^{6} \mathrm{~S}-\mathrm{a}^{4} \mathrm{P}$ being probably the strongest $[C r \mathrm{II}]$ transition, or at least one of the strongest. The predicted wave lengths of the $a^{6} S-a^{4} P$ lines are given in the following table.

$\begin{array}{cc}\mathrm{a}^{6} \mathrm{~S}-\mathrm{a}^{4} \mathrm{P} & \text { LiNes of }[C r \text { II }] \\ \text { Transition } & \lambda \text { Predicted } \\ 5 / 2-5 / 2 & 4581.18 \\ 5 / 2-3 / 2 & 4580.80 \\ 5 / 2-1 / 2 & 4580.88\end{array}$

The mean wave length of the predicted multiplet is $\lambda 4581.1$ $\pm 0.1 \mathrm{~A}$.

$\eta$ Carinae seemed to be the most promising star in which to look for possible [ $\mathrm{Cr}$ II]. In their list of wave lengths in the spectrum of $\eta$ Carinae, Moore and Sanford ${ }^{1}$ do not give any emission line immediately to the violet of the strong $F e$ II line $\lambda 4583.8$, but Lunt ${ }^{2}$ gives a line of intensity 1 at $\lambda 4580.36$. Thanks to the courtesy of Drs. Moore and Sanford, I was given an opportunity to re-examine the original spectrogram of $\eta$ Carinae obtained at the Lick Observatory station in Chile. I found a line definitely present to the violet of the $F e$ II line $\lambda 4583.8$.

A series of settings made by Dr. Merrill on the unidentified line and on the $F e$ II lines at $\lambda 4576.33$ and $\lambda 4583.83$ gave by interpolation the wave length $\lambda 4581.12$, which agrees perfectly with the predicted $[\mathrm{Cr}$ II] line. No other satisfactory identification can be found for the new line.

The presence of $[\mathrm{Cr}$ II] in stars with $[\mathrm{Fe}$ II] had been suggested previously ${ }^{3}$ on the basis of a coincidence between the wave length $\lambda 3993.1$ of a line observed in WY Geminorum and in B 1985 and the predicted wave length $\lambda 3993.57$ of the $a^{6} \mathrm{~S}_{5 / 2}-$ $b^{4} D_{7 / 2}$ transition; the wave lengths predicted for the other transi-

${ }^{1}$ Lick Obs. Bull., 8, 55, 1913.

2 M.N., 79, 621, 1919.

${ }^{3}$ Swings and Struve, $A p$. J., 91, 600, $1940 ;$ 93, 457, 1941. 
tions of the same multiplet are respectively $3993.29(5 / 2-1 / 2)$, $3992.08(5 / 2-3 / 2)$, and $3991.47(5 / 2-5 / 2)$. H. Spencer Jones lists a line in $\eta$ Carinae $^{4}$ at $\lambda$ 3992.9. This line may be due to $[C r \mathrm{II}]$, but the predicted [Ni II] line $\mathrm{a}^{2} \mathrm{D}_{5 / 2}-\mathrm{a}^{4} \mathrm{P}_{5 / 2}$ falls also at $\lambda$ 3993.3, and other [NiII] lines are known to be present in $[\mathrm{Fe}$ II] stars. Hence $[\mathrm{Cr}$ II] could not be identified with certainty on the basis of the $a^{6} \mathrm{~S}-\mathrm{b}^{4} \mathrm{D}$ transition alone. The line observed at $\lambda 4581.1$ in $\eta$ Carinae provides much better evidence. It is interesting to note that the $F e$ Ir line $\lambda 4583.8$ is thus accompanied on the violet side by a forbidden $C r$ II line $(\lambda$ 4581.1) and on the red side by a permitted $C r$ II line $(\lambda 4588.2)$.

The following [NiII] transitions have been observed: ${ }^{5}$ $\mathrm{a}^{2} \mathrm{D}-\mathrm{a}^{2} \mathrm{P}$ (strongest line at $\left.\lambda 3439.1\right) ; \mathrm{a}^{2} \mathrm{D}-\mathrm{a}^{2} \mathrm{G}$ ( $\lambda$ 3223.6), $\mathrm{a}^{2} \mathrm{D}-\mathrm{b}^{2} \mathrm{D}(\lambda 4326.5$ and $\lambda 4201.4)$, and possibly one or two others. The ground level is $\mathrm{a}^{2} \mathrm{D}$; the excited levels providing the strong ultraviolet lines have the following excitation potentials:

$$
\mathrm{a}^{2} \mathrm{P}_{3 / 2}=3.60 \text { volts ; } \mathrm{a}^{2} \mathrm{G}_{9 / 2}=4.03 \text { volts. }
$$

$N i$ Ir has a level $\mathrm{a}^{2} \mathrm{~F}$ at 1.68 volts, hence 1.92 volts lower than $\mathrm{a}^{2} \mathrm{P}_{3 / 2}$. The ratio $r$ of the populations on the $\mathrm{a}^{2} \mathrm{~F}_{7 / 2}$ and $\mathrm{a}^{2} \mathrm{P}_{3 / 2}$ levels is immediately obtained by the Boltzmann relation

$$
r=\frac{8}{4} \times e^{\frac{11600 \times 1.92}{T}} \text {. }
$$

In this expression, $T$ is the electron temperature which is known to be low in shells giving forbidden lines of singly ionized metals. Adopting

it is found that

$$
T=6000^{\circ} \text {, }
$$

$$
r=82 .
$$

If, as is most likely, $\mathrm{a}^{2} \mathrm{D}-\mathrm{a}^{2} \mathrm{~F}$ has a transition probability similar to that of $a^{2} \mathrm{D}-\mathrm{a}^{2} \mathrm{P}$, the red multiplet $\mathrm{a}^{2} \mathrm{D}-\mathrm{a}^{2} \mathrm{~F}(\lambda \lambda 6667,7378$, $7412,8302)$ must be extremely strong relative to the ultraviolet multiplet $a^{2} \mathrm{D}-\mathrm{a}^{2} \mathrm{P}(\lambda \lambda 3378,3439,3560,3627)$, the intensity

\footnotetext{
${ }^{4}$ M.N., 91, 794, 1931.

5 P. Swings, Pub. A.S.P., 55, 276, 1943.
} 
ratio being 82 times the wave-number ratio, ${ }^{6}$ or approximately 40. Since $\lambda 3439$ reaches a fairly high intensity in certain $\mathrm{Be}$ stars we may thus expect the red multiplet $a^{2} \mathrm{D}-\mathrm{a}^{2} \mathrm{~F}$ to be an outstanding feature in the same stars.

Weak bright lines, probably stationary, have been observed in $v$ Sagittarii at $\lambda 7155.1$ and $\lambda 7711.5$ by P. W. Merrill. ${ }^{7}$ The first line coincides exactly in wave length with the $[F e$ II] line, $\mathrm{a}^{4} \mathrm{~F}_{9 / 2}-\mathrm{a}^{2} \mathrm{G}_{9 / 2}, \lambda$ pred. 7155.1. If this transition $\mathrm{a}^{4} \mathrm{~F}_{9 / 2}-\mathrm{a}^{2} \mathrm{G}_{9 / 2}$ has a probability of the same order as that of the violet [ $\mathrm{Fe}$ II] multiplets $a^{6} \mathrm{D}-\mathrm{a}^{6} \mathrm{~S}$ or $\mathrm{a}^{6} \mathrm{D}-\mathrm{b}^{4} \mathrm{~F}$, the $\lambda 7155.1$ line should be very strong relative to the violet $[F e \mathrm{II}]$ lines, since the $\mathrm{a}^{2} \mathrm{G}$ level is much lower than $\mathrm{a}^{6} \mathrm{~S}$ or $\mathrm{b}^{4} \mathrm{~F}$. This can be proven observationally as well as theoretically as follows.

The strongest lines of the $\mathrm{a}^{4} \mathrm{P}-\mathrm{z}^{6} \mathrm{P}^{0}$ permitted multiplet at $\lambda 3938$ and $\lambda 3914$ (E.P. of upper level 4.8 volts) which are very weak in the laboratory (int. 2) appear fairly strongly in emission in certain $\mathrm{Fe}$ II stars. On the other hand a line of laboratory intensity 3 in the $b^{4} P-z^{4} D^{0}$ multiplet (E.P. of upper level 5.5 volts) which contains very intense lines is extremely weak in emission in the stars where $\lambda 3938$ is fairly strong. An increase in excitation potential of 0.7 volt suffices thus to bring about a considerable decrease in intensity of the emission lines. The same intensity relationships would hold for the $[\mathrm{Fe}$ II $]$ lines.

Moreover the same reasoning used for [NiII] can be repeated here. If we adopt an electron temperature of $6000^{\circ}$ and assume again a pure collisional excitation, the populations on the levels $\mathrm{a}^{2} \mathrm{G}_{9 / 2}$ and $\mathrm{a}^{6} \mathrm{~S}_{5 / 2}$ turn out to be in the ratio of 10 to 1 .

The identification of an infrared [ $F e$ II] line of low excitation potential is thus permissible even when none of the usual violet $[F e \mathrm{II}]$ lines is observed. As a general rule, any metallic element will have stronger forbidden lines in the infrared than in the violet because, in a statistical way, the infrared lines correspond to excitation energies lower than those of the violet lines.

6 This figure gives, of course, only an order of magnitude, since we have neglected various effects, such as collisional de-excitation.

${ }^{7}$ Pub. A.S.P., 55, 42, 1944, and information kindly given by Dr. Merrill. 
The upper level of the [CaII] lines identified by Merrill in $v$ Sagittarii has a very low energy.

The line observed by Merrill at $\lambda 7711.5$ falls near the predicted [Fe II] line, $\mathrm{a}^{4} \mathrm{D}_{1 / 2}-\mathrm{b}^{4} \mathrm{P}_{3 / 2}$ at $\lambda 7710.8$, but the agreement in wave length is not satisfactory. Moreover $\mathrm{b}^{4} \mathrm{P}$ is a fairly high level (E.P. 2.69 volts), and the identification of $\lambda 7711.5$ with [ $F e$ II] would thus require the presence of the violet [ $F e$ II] lines. The line 7711.5 of $v$ Sagittarii still remains unidentified. Another $[F e$ II $]$ multiplet $\mathrm{a}^{6} \mathrm{D}-\mathrm{a}^{4} \mathrm{D}$, near $\lambda 12,000$, may be expected to be very intense in all [Fe II] stars since the excitation potential of $\mathrm{a}^{4} \mathrm{D}$ is only about 1 volt.

\section{Pasadena, California}

October 19, 1944

\section{FIRST ASTRONOMICAL PHOTOGRAPHS FROM THE MORSE OBSERVATORY}

Pvt. Stuart J. Inglis, USMCR

As part of a course in practical astronomy at Occidental College some pictures of the moon were taken with the 15 -inch (f/6.4) reflector recently given to the College by Mrs. Edward H. Morse. ${ }^{1}$

After several trials with a plateholder replacing the eyepiece at the Newtonian focus, it was decided to photograph at the prime focus. This necessitated removing the Newtonian flat and suspending a camera eight inches outside the upper end of the tube. In the machine shop at the College, I built a simple mounting which can be fastened to the tube to support the plateholder in the required position.

The accompanying picture of the moon (Plate XIV) was obtained on October 25, 1944, at approximately 10 P.M., P.W.T. It shows the moon about one day after first quarter. With Eastman Super Pancro-Press Type B emulsion the exposure time was 0.1 second.

I wish to express my appreciation to Dr. Gerard F. W. Mulders under whose guidance my work in astronomy was done.

\footnotetext{
${ }_{1}$ Pub. A.S.P., 56, 97, 1944.
} 